\title{
Anticancer activity of the seaweed compound fucoxanthin in breast cancer cell lines cultured as 2D and 3D models
}

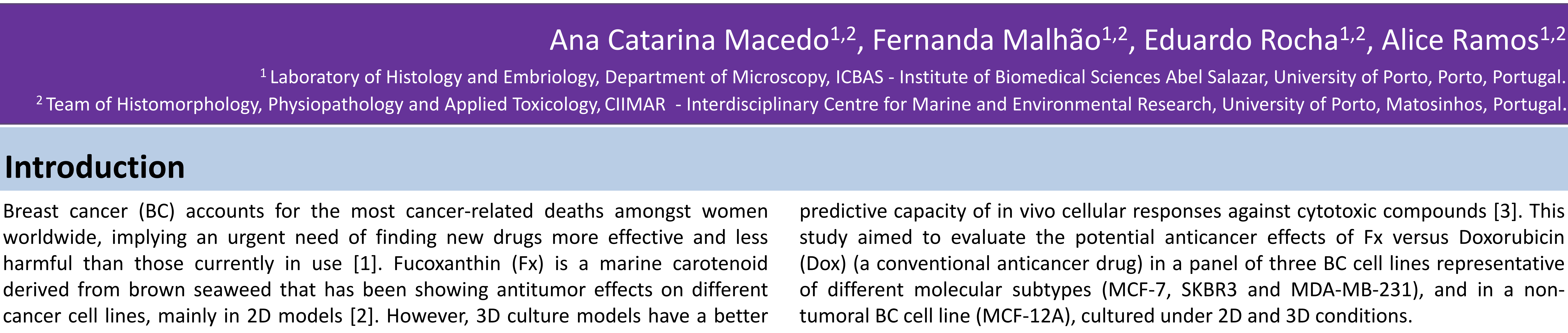

\section{Results and Discussion}

\section{D cultures}

Effects on cell viability and death

$\rightarrow$ Fx and Dox induced cytotoxic effects on all the cell lines tested.
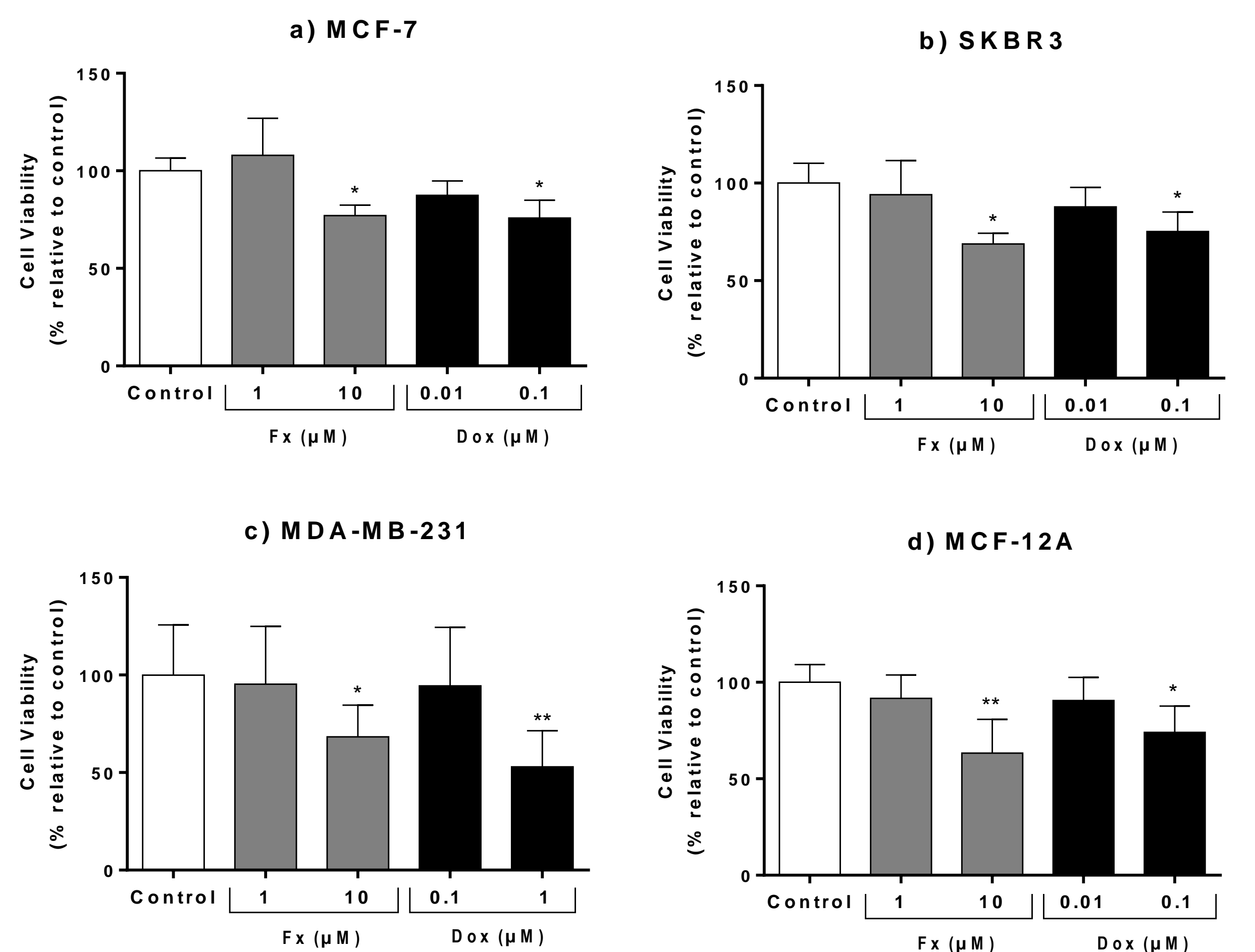

Figure 1- Effect of Fx and Dox on cell viability in MCF-7 (a), SKBR3 (b), MDA-MB-231 (c) and MCF12A (d) cell lines assessed by the MTT assay Cells treated with $0.1 \%$ DMSO were included as negative control. Values are presented as mean $+S D$ of at least three independent experiments. Significant differences $\mathrm{P} \leq 0.05$ and ${ }^{* *} \mathrm{P} \leq 0.01$ ) when compared with control were tested by one-way ANOVA, followed by posthoc Holm-Sidak's multiple comparison test.

$\rightarrow$ Dox increased cell death in MCF-12A cells.

$\rightarrow$ No cell death or genotoxic effects registered

in Fx-treated cells.

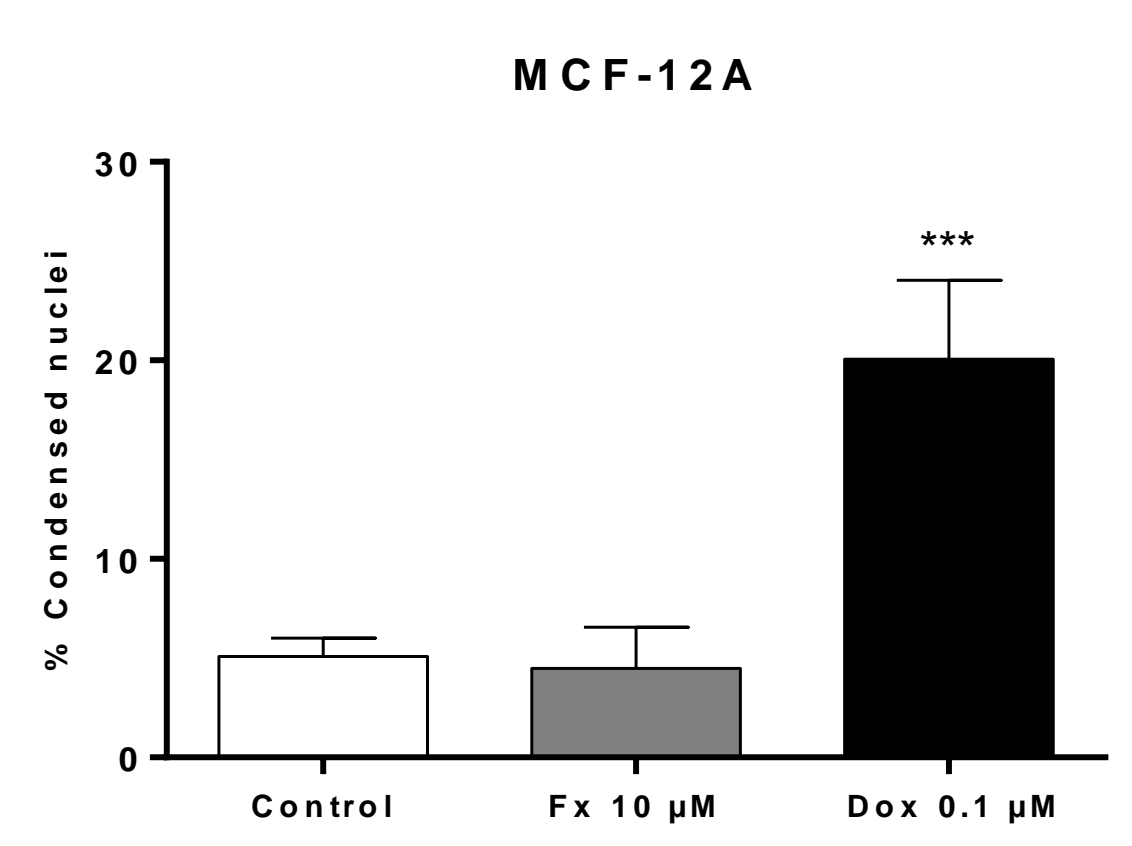

Figure 2- Effects of $\mathrm{Fx}(10 \mu \mathrm{M})$ and Dox $(0.1 \mu \mathrm{M})$ on cell death in MCF-12A cells assessed by the nuclear condensation assay. Cells treated with $0.1 \%$ DMSO were included as negative control. Values are presented as mean $+S D$ of at least three independent experiments. Significant differences $(* * * P \leq 0.001$ and $* * * * P \leq 0.0001$ ) when compared with control were tested by one-way ANOVA, followed by post-hoc Holm-Sidak's multiple comparison test.

\section{Experimental methodology}

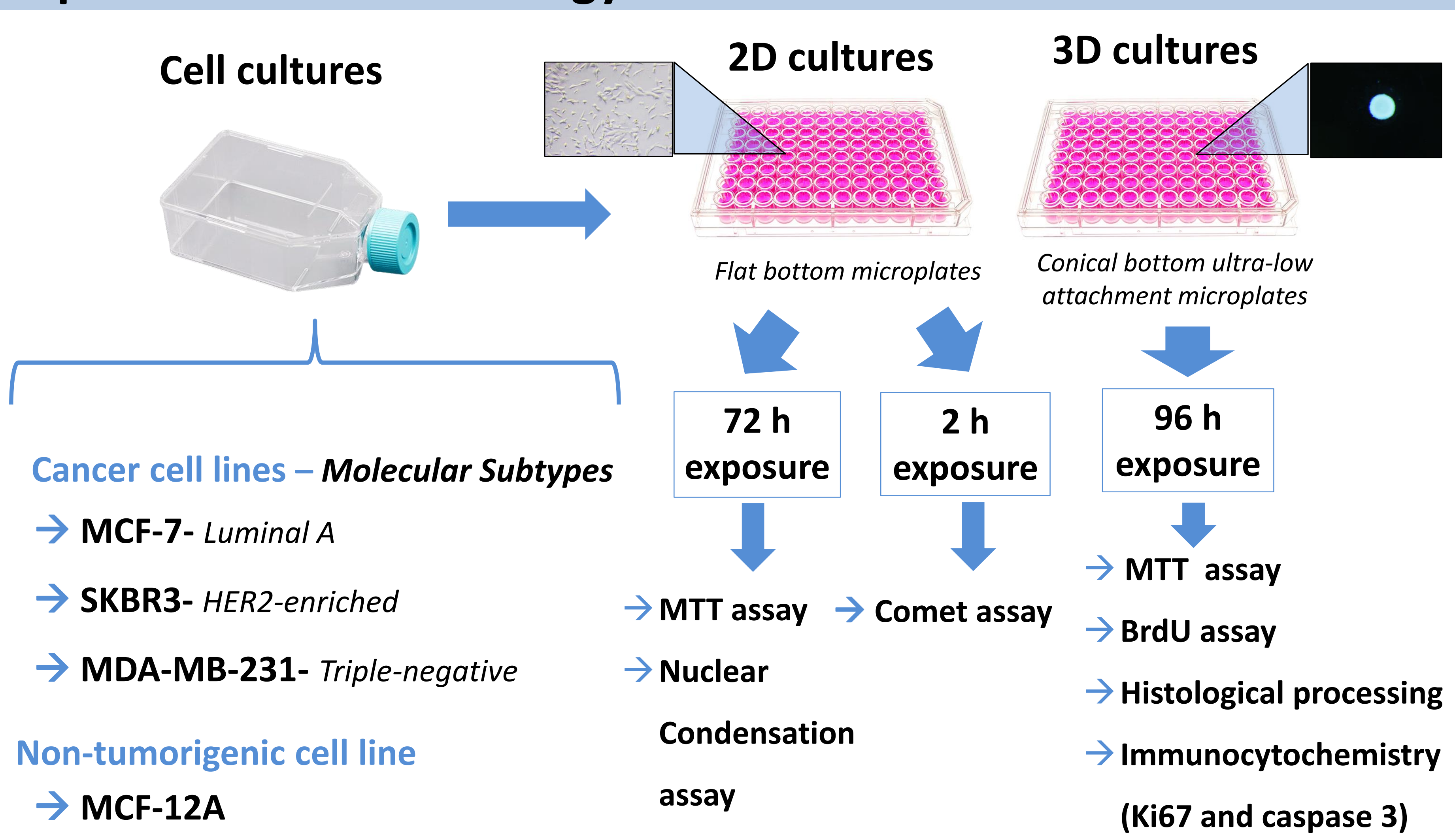

\section{Acknowledgements}

We thank the fundamental financial support of the ICBAS of the UPorto, namely via its Laboratory of Histology and Embryology and Master Program in Environmental Contamination and Toxicology. This research was also partially supported by the Strategic Funding UID/Multi/04423/2019 through national funds provided by FCT and ERDF, in the framework of the program PT2020. We also thank the team of analysis of the comet assay data.

\section{D cultures}

Effects on cell viability, proliferation and death

$\rightarrow$ In 3D, cells were less responsive to Fx and Dox (no cytotoxic effects).
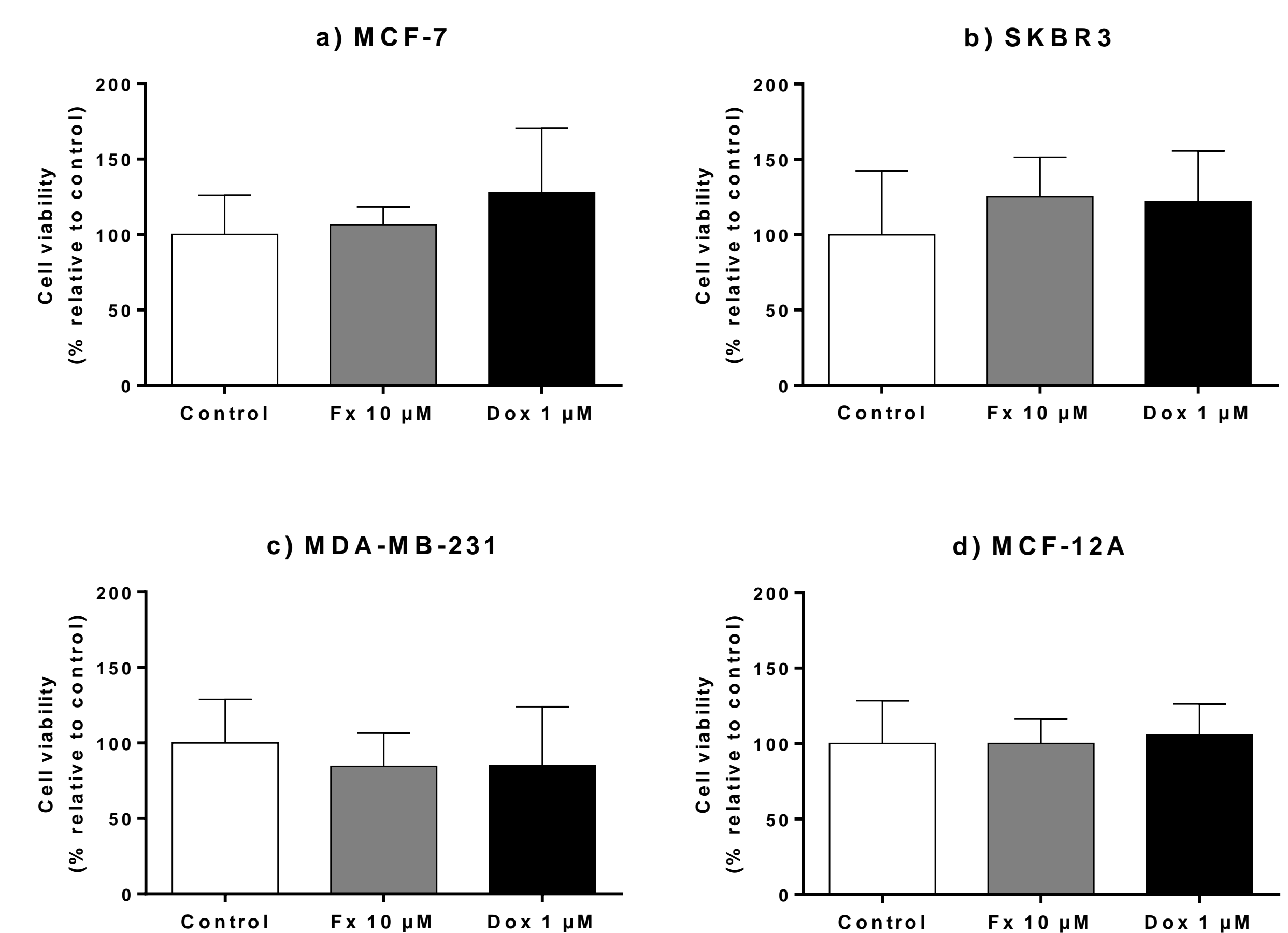

Figure 3- Effect of $\mathrm{Fx}(10 \mu \mathrm{M})$ and Dox (1 $\mu \mathrm{M})$ on the viability of MCF-7 (a), SKBR3 (b), MDA-MB-231 (c) and MCF12A (d) cell lines assessed by the MTT assay. Cells treated with $0.1 \%$ DMSO were included as negative control. Values are presented as mean + SD of at least four independent experiments. No significant effects were detected by one-way ANOVA analysis.

$\rightarrow$ Dox affected the proliferation of SKBR3 and MCF-12 A cell lines. Also, there were less Ki67 positive cells in SKBR3 3D cultures treated with Dox.

$\rightarrow$ Fx only caused antiproliferative effects on the SKBR3 3D models.
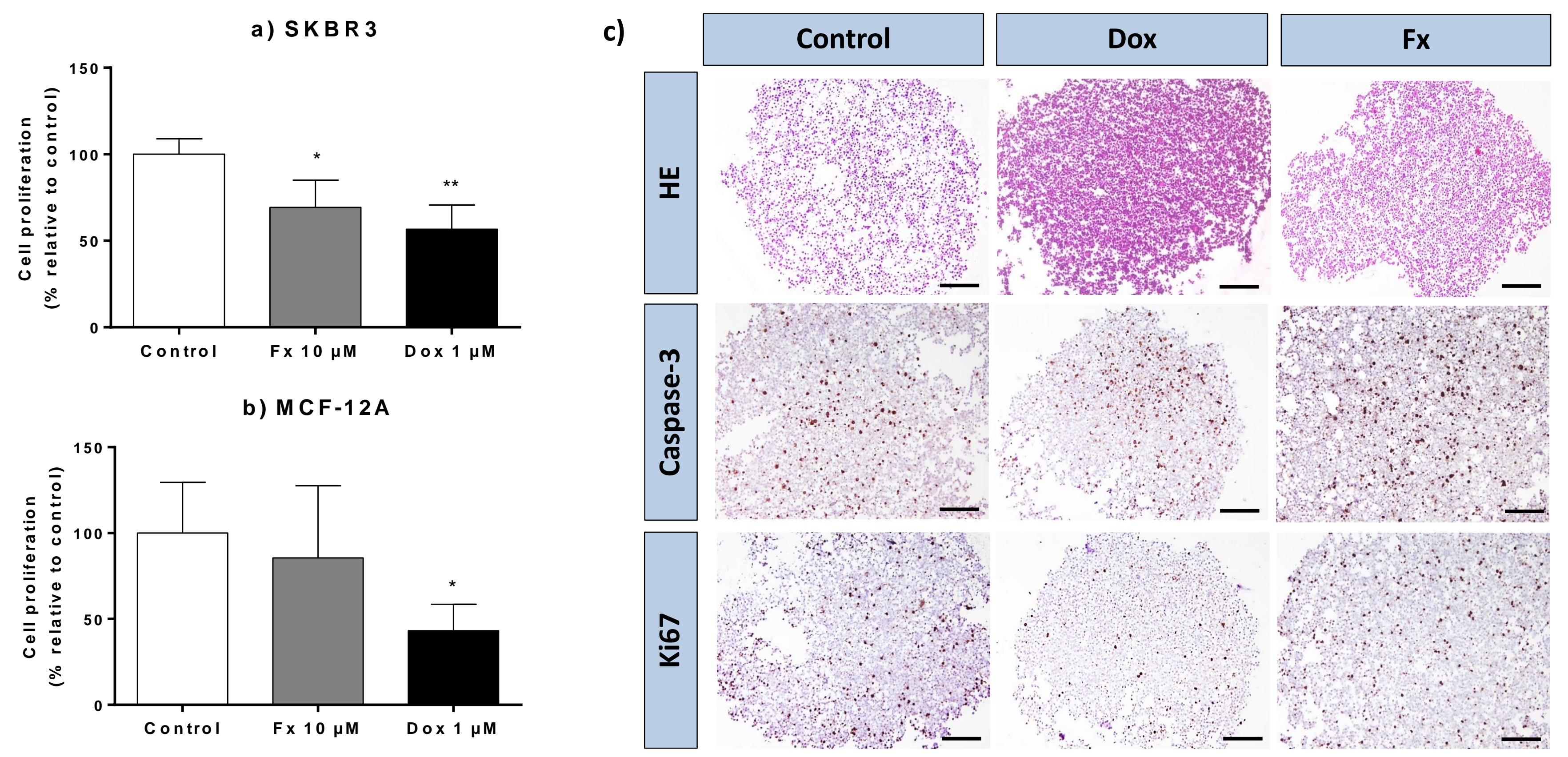

Figure 4- Effect of $F x(10 \mu M)$ and Dox $(1 \mu \mathrm{M})$ on cell proliferation of SKBR3 (a) and MCF-12A (b) cell lines, assessed by the BrdU assay. Cells treated with 0.1\% DMSO were included as negative control. Values are presented as mean $+S D$ of four independent experiments. Significant differences $*^{*} p<0.05$ and ${ }^{* *} p<$ 0.01 ) when compared to the control were determined by one-way ANOVA, followed by a Holm-Sidak's multiple comparisons test. Representative sections (c) of the SKBR3 3D models stained with haemotoxylin and eosin (HE) and immunostained against caspase-3 and Ki67 (brown staining) to assess cell death and proliferation, respectively. Scale bar: $200 \mu \mathrm{m}$.

\section{Conclusion}

The in vitro data revealed that Fx may be a potential anticancer agent against BC cells, with differential effects according to the cell subtype. Also, the comparison between the two cell culture models indicated that cells' resistance towards Fx and Dox anticancer activity increased under 3D conditions. The data warrants further studies on the underlying anticancer mechanisms.

\section{References}

[1]- DeSantis, C.E. Bray, F. Ferlay, J. et al. (2015). Cancer Epidemiol Biomarkers Prev 24, 1495-506. DOI: 10.1158/1055-9965.EPl-15-0535 [2]-Kumar, S.R. Hosokawa, M. and Miyashita,K. (2013). Mar Drugs 11, 5130-47. DOI: 10.3390/md11125130
[3]-Santo, V.E. Rebelo, S.P. Estrada, M.F. et al. (2017). Biotechnol J 12, 1600505. DOl: 10.1002/biot.201600505 